\title{
DISEÑO Y EVALUACIÓN DE SISTEMAS DE POLINIZACIÓN ENTOMOLÓGICA A (Passiflora edulis) EN EL VALLE DEL MAGDALENA, TOLIMA
}

\author{
Ricardo Tello ${ }^{90}$
}

RESUMEN

El maracuyá Passiflora edulis es una fruta que se utiliza en fresco para la preparación de jugos, helados o en la agroindustria como materia prima de un sin número de productos. En los últimos dos años en la zona del Valle del Magdalena en los municipios de El Espinal, Guamo y Coello se han instalado cerca de 500ha, del cultivo de maracuyá utilizando la tecnología desarrollada en el Valle del Cauca, esto ha generando ocupación de mano de obra importante para la economía de la región.

Sin embargo, factores como el clima, los suelos y la condición de auto esterilidad del maracuyá, han causado problemas en los flujos de caja de los cultivos por la baja producción. Por lo tanto, se ha requerido la polinización manual de los cultivos buscando incrementar el volumen de producción pero se ha observado que la eficiencia de polinización es baja, lo que causa poca formación de frutos o que se formen con bajo peso, además de los sobre costos que ello implica.

La información científica está disponible y observaciones de campo conducen a que los mejores sistemas de polinización del maracuyá, debe ser con insectos (entomología). Al hacer la revisión de la literatura, al respecto, en fuentes primarias y secundarias se determinó que la abeja carpintera del género Xilocopa lachnea y la abeja africanizada Apis mellifera, son los polinizadores naturales del cultivo.

Por lo tanto, se diseñaron dos sistemas de polinización con dichos insectos para compararlos con el método manual y se evaluarán eficiencia de polinización, calidad de frutos, peso y costos de implementación y manejo de cada sistema para incrementar la productividad del maracuyá en la región.

Palabras clave: auto esterilidad, polinización entomológica, producción.

\begin{abstract}
The passion fruit is used as a prime material for different products. For the last two years, about 500 Ha of crops have been harvested in the Magdalena Valley, an important source of work for the region people.
\end{abstract}

90 Ing. Agrónomo. Tutor CEAD Ibagué, integrante grupo de Investigación GIEPRONAL. Escuela de Ciencias Agrícolas Pecuarias y del Medio Ambiente, ECAPMA, UNAD. 
However, the passion fruit has a natural condition of auto sterilization. Manual polinization is needed. The efficiency is too low because the crops become too small. This scientific information points to the entomologic polinization systems, which are best for the passion fruit. Also the scientists confirm that the natural polinization of the crops come from both the carpenter bee (Xilocopa lachnea) and African bee (Apis mellifera).

This project utilizes two entomologic polinization systems in order to compare the manual polinization. It will be evaluate the efficiency of polinization, the crop's quality and weight, as well as management costs.

The two entomologic polinization systems will be located in El Espinal and In Guamo (both cities of Tolima Department) about 10 Ha passion fruit crops each area. Also provide with a dispersion center which is a cube 4 meters edge, cover with black net, hermetic in order to control de insects and its flowers access.

The methodology depends of the species and the biological cycle of the passion fruit flower. For the carpenter bees, it will be necessary to keep the humidity through a drop water system and feeding supplies. For the African bees, be recollected the pollen will be recollected and sprayed afterwards during the opening flower.

When the polinization is successfull, the passion fruit flower will be marked in order to evaluate the becoming crops with the parameters such as shape, size, color and weight, and compare these parameters with those that used manual polinization. The parameters will be taken in a 90 day period after the adaptation period of the colonies finishes, in each case about 20 days.

The goal of this project is to evaluate the three polinization methods for a profitable and sustainable agriculture. This tries to find a better harvest of passion fruit that contributes to crops improvement, and environmental impact. Humanity has abused the use of agrochemicals and bees, so we have to fix this before it is too late.

Key words: Auto Sterility, Pollination, Entomological Pollination, Production.

\section{INTRODUCCIÓN}

El departamento del Tolima se ha consolidado como productor de alimentos que surte a la capital de la República. Los cambios en los hábitos alimenticios de la población han generado una creciente demanda de frutas lo que ha originado la especialización de regiones agrícolas del Valle del Magdalena, que cuentan con suministro de agua de distritos de riego, infraestructura de acceso, servicios públicos y mano de obra.

En los años 2009-2010 se han sembrado cerca de 500 hectáreas de Maracuyá Passiflora edulis, en la zona plana de los municipios del Guamo, Coello y El Espinal que presentan problemas de bajos rendimientos por la baja polinización de las flores ya que el maracuyá 
es auto - estéril, y los polinizadores naturales Xilocopa lachnea y Apis mellifera, no se encuentran en cantidades adecuadas para el cultivo intensivo.

El presente trabajo busca evaluar tres métodos de polinización que hagan rentable y sostenible el cultivo.

\section{OBJETIVOS GENERAL}

Evaluar el sistema de polinización entomológica de passiflora edulis en el Valle del Magdalena, Departamento del Tolima.

\section{OBJETIVO ESPECÍFICOS}

Diseñar el sistema de polinización de la especie abeja carpintera del género Xilocopa lachnea para el cultivo del maracuyá Passiflora edulis.

Diseñar el sistema de polinización asistida con abejas del género Apis mellifera, para el cultivo del maracuyá Passiflora edulis.

Evaluar técnica y económicamente cada sistema comparado con el sistema de polinización manual.

\section{METODOLOGÍA}

Para realizar la evaluación de los sistemas de polinización se instalarán parcelas de 2 ha, en predios ubicados en la zona del valle del Magdalena en los municipios del El Espinal y Guamo, para lo cual se identificaron lotes del mismo tamaño de cultivo de maracuyá en producción, cada parcela está alejada de la siguiente $200 \mathrm{~m}$ para evitar la influencia de un tratamiento en el otro. Los insectos se instalarán cerca a los cultivos, dentro de "Centros de dispersión". Dichos centros están construidos en forma de cubo de 4 metros de arista, cubierto por una malla de color negro, herméticos para controlar la salida de los insectos y el acceso a las flores en los momentos deseados y permitir que se realicen las aplicaciones de agroquímicos al cultivo sin detrimento de la población de insectos. La metodología del manejo de las colonias de cada especie es la siguiente:

\section{Tratamiento 1: polinización con Abeja Carpintera Xilocopa lachnea}

Observando los hábitos de nidificación de la abeja carpintera Xilocopa lachnea se determinó que en troncos secos construyen sus nidos y viven con sus crías, las cuales alimentan con néctar y polen; forman colonias aunque en cada orificio habitan individualmente. Para el presente estudio se recuperarán colonias silvestres originarias del Valle del Magdalena y se ubicarán en el centro de dispersión de polinizadores, localizado en el cultivo de maracuyá. Las abejas carpinteras se protegerán de los rayos solares, vientos fuertes, lluvia y depredadores naturales, se incrementará la humedad relativa del "Centro de Dispersión" mediante la adición controlada de agua con un sistema de riego por goteo, también se suministrará 
alimento constituido por miel y polen colectado por Apis mellifera, en cantidad suficiente, para permitir la rápida reproducción de la abeja carpintera.

La flor de maracuyá Passiflora edulis realiza apertura a las 11 am hasta las 5 pm en días soleados, horas en las cuales se debe permitir que los polinizadores tengan acceso a las flores, en estos momentos ocurre la mayor producción de néctares y su aroma es un potente atrayente para los insectos, del mismo modo ocurre la dehiscencia del polen de las anteras, además, los estigmas están receptivos.

Para realizar la evaluación se marcarán las flores visitadas, y los frutos allí formados, se le evaluarán parámetros como forma, tamaño, color y peso y se compararán con frutos que se originan de lotes con polinización manual y con el tratamiento 2.

\section{Tratamiento 2: polinización asistida con abeja africanizada Apis mellifera}

Mediante la instalación de colmenas convencionales, tipo Lanstrong de 10 cuadros con trampa de polen, se pretende colectar polen de maracuyá con abejas africanizadas, induciéndolas a visitar las flores mediante la alimentación artificial de jarabe con jugo de maracuyá.

El polen colectado se llevará al laboratorio para macerarlo y diluirlo en agua potable con 10\% de jarabe de azúcar y luego asperjarlo a las flores durante su apertura, horario del que ya se habló. De esta manera se asegura la cantidad de polen en los estigmas receptivos.

Las colmenas se alimentaran dos veces a la semana con jarabe de azúcar al 50\% enriquecido con $10 \%$ de miel de abejas. Para realizar la toma de datos de las flores asperjadas con polen diluido, se marcarán y a los frutos allí formados se le evaluarán parámetros como son: forma, tamaño, color y peso. Dichos resultados se compararán con frutos que se originan de lotes con polinización manual y con los obtenidos con polinización de abeja carpintera.

\section{Tratamiento 3: polinización manual}

Corresponde al sistema que se está realizando con mano de obra local, se identificarán las parcelas testigo y se les realizará a las flores y frutos, el mismo seguimiento que en los tratamientos anteriores.

Obtenidos los resultados se tabularán y utilizando un diseño experimental de bloques al azar, con tres tratamientos y la unidad experimental en la parcela de 1ha, eliminando los bordes $(10 \mathrm{~m})$ para reducir el margen de error, se pretende determinar cuál tratamiento produce los mejores frutos. La toma de datos se realizará durante 90 días ininterrumpidos, una vez terminado el proceso de adaptación de los insectos antes mencionado.

De la misma manera, se evaluarán los costos de cada tratamiento y se determinará cuál es el tratamiento o sistema de polinización más competitivo para el cultivo de maracuyá en el valle del Magdalena. 


\section{RESULTADOS PARCIALES}

El proyecto se encuentra en la etapa de instalación y adaptación de los insectos al sitio, buscando que factores como transporte, manejo y alimentación no interfieran en el potencial polinizador de los mismos. Antes de comenzar a realizar evaluaciones se estima que el tiempo de adaptación es de 20 días.

Sin embargo, una vez instalados en los “centros de dispersión" los dos agentes polinizadores estudiados, se pudo observar que sí visitan las flores que están abiertas y que tanto la abeja carpintera, como la africanizada se alimentan y alimentan a sus crías con polen y néctar de las flores de maracuyá.

Con las observaciones y la experiencia del manejo de la abeja africanizada se espera realizar la cría y manejo de la abeja carpintera, que si bien es cierto que es común encontrarla en el Valle del Magdalena, no se ha domesticado para utilizarla en polinización dirigida a cultivos comerciales.

\section{PERSPECTIVAS DEL TRABAJO}

La utilización de insectos para realizar polinización asistida de cultivos comerciales tiene gran importancia en los países desarrollados y se estima que el 30\% de los productos que tenemos en nuestra dieta requieren la polinización entomológica para su producción.

En el Valle del Magdalena se utiliza la polinización dirigida en melón de forma comercial y se están realizando trabajos de investigación por parte de Corpoica y la UN en mango (Chicoral), aguacate (fresno), Mora (Icononzo), en el departamento del Tolima.

En el caso del maracuyá Passiflora edulis el presente trabajo busca dar una solución a un problema grave: la baja producción de frutos que se obtiene en la actualidad bajo el sistema de polinización manual; se espera que el cultivo instalado en la región de estudio, aproximadamente $500 \mathrm{Ha}$, sea altamente productivo e induzca a la siembra de nuevas áreas, generando empleos permanentes.

El maracuyá es un cultivo semipermanente con un ciclo de vida que dura dos años, esto permite la disminución de utilización de maquinaria y agroquímicos en los lotes establecidos y mitiga el impacto ambiental causado por la agricultura convencional de cultivos semestrales.

\section{DISCUSIÓN}

Los inversionistas agropecuarios han unido esfuerzos para cambiar de cultivos tradicionales mecanizados y baja rentabilidad a cultivos de frutas que exigen mayores capitales, mayor tecnología, capacitación y entrenamiento continuo a los operarios, aparentemente presenta mayor rentabilidad y estabilidad de precios y mercado asegurado en los mercados mayoristas de los centros urbanos especialmente la capital de la República. Estos cultivos demandan 
mayor atención y administración por parte de los agricultores que en la mayoría de los casos son residentes en Santa fe de Bogotá.

El maracuyá es una planta de polinización cruzada, auto compatible pero auto estéril, la transmisión del polen puede realizarse a través del viento, siendo la más eficiente la realizada por medio de insectos porque las flores son grandes, atractivas, con abundante aroma y néctar, los granos de polen son grandes y pegajosos. La polinización depende principalmente de los insectos, la humedad del estigma y la curvatura del estilo. De este tipo de polinización depende en gran parte la fructificación.

Los productores de maracuyá Passiflora edulis tienen grandes expectativas con el desarrollo del proyecto, puesto que las bajas producciones hacen que la rentabilidad del cultivo sea poca y desestimula la siembra de áreas nuevas. El proyecto pretende coadyuvar en la que de forma natural ocurre cuando las poblaciones de insectos son adecuadas y el uso de agroquímicos es racional. En el área de estudio durante varias décadas se ha abusado del uso de agroquímicos y la fauna benéfica de polinizadores naturales es muy escasa, por lo tanto, debemos mediante métodos ecológicamente viables solucionar un problema que causó el hombre al realizar malas prácticas agrícolas a través del tiempo.

Se espera que con el desarrollo del proyecto se determine el sistema de polinización más competitivo, sostenible y ecológicamente viable.

\section{CONCLUSIONES}

El maracuyá es un cultivo promisorio para el Valle del Magdalena, con buenos precios de mercado y demanda creciente en los centros urbanos.

Presenta un problema de baja producción por falta de polinización natural ya que su condición de auto esterilidad dificulta la llegada del polen al estigma.

A los polinizadores naturales del maracuyá se les debe dar las condiciones adecuadas de manejo para permitir su establecimiento junto con el cultivo, permitiendo realizar la su relación biológica de ayuda mutua (vegetal-insecto).

La UNAD juega un papel importantísimo al buscar soluciones reales a problemas reales y actuales del agro colombiano y de la región.

La integración de semilleros de investigación, grupos de investigación y la comunidad hace que el la Universidad Nacional Abierta y a Distancia actúe como un nodo articulador productor de conocimiento.

Estos proyectos abren puertas a semilleros y estudiantes para realizar nuevas investigaciones y hacen conocer el trabajo de los grupos de investigación con la comunidad. 


\section{REFERENCIAS BIBLIOGRÁFICAS}

DA SILVA, Airon M, BRUCKNER, Claudio H, PICANCO, Marcelo et al. Número floral, clima, densidad poblacional de Xylocopa spp. (Hymenoptera: Anthophoridae) y polinización del maracuyá (Passiflora edulis f. flavicarpa). Rev. biol. trop, dic. 1999, vol.47, núm.4, p.711-718. ISSN 0034-7744.

DA SILVA, Airon M, BRUCKNER, Claudio H, PICANCO, Marcelo et al. Número floral, clima, densidad poblacional de Xylocopa spp. (Hymenoptera: Anthophoridae) y polinización del maracuyá (Passiflora edulis f. flavicarpa). Rev. biol. trop. [online]. dic. 1999, vol.47, núm.4 [citado 13 abril 2010], p.711-718. Disponible en la World Wide Web: <http://www.scielo.sa.cr/scielo.php?script=sci_arttext\&pid=S003477441999000400007\&lng=es\&nrm=iso>. ISSN 0034-7744.

ROBERT J, KNIGHT, Jr. , Julian W. Sauls, Carlos F. Balerdi y Jonathan H. Crane2 El Maracuyá o Parchita en Florida1, U.S. Department of Agriculture, Cooperative Extension Service, University of Florida, IFAS, Florida A. \& M. University Cooperative Extension Program, and Boards of County Commissioners Cooperating. Millie Ferrer-Chancy, Interim Dean.

VÁSQUEZ, Rodrigo, TELLO, Jorge E. CORPOICA, UNIVERSIDAD NACIONAL. Evaluación de polinización de Apis mellifera en mango, aguacate, mora, fresa y cítricos. 2009. 